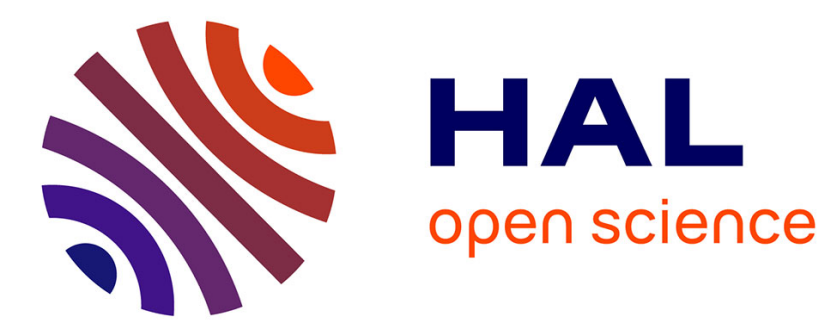

\title{
Le rapport au temps, à l'espace et au discours des voix d 'un point de vue psychopathologique
} Ingrid Guigo-Banovic, Guy Gimenez, Jean-Louis Pedinielli

\section{To cite this version:}

Ingrid Guigo-Banovic, Guy Gimenez, Jean-Louis Pedinielli. Le rapport au temps, à l'espace et au discours des voix d 'un point de vue psychopathologique. Annales Médico-Psychologiques, Revue Psychiatrique, 2005, 163, pp.726-731. 10.1016/j.amp.2004.04.021 . hal-01383869

\section{HAL Id: hal-01383869 \\ https://hal-amu.archives-ouvertes.fr/hal-01383869}

Submitted on 19 Oct 2016

HAL is a multi-disciplinary open access archive for the deposit and dissemination of scientific research documents, whether they are published or not. The documents may come from teaching and research institutions in France or abroad, or from public or private research centers.
L'archive ouverte pluridisciplinaire HAL, est destinée au dépôt et à la diffusion de documents scientifiques de niveau recherche, publiés ou non, émanant des établissements d'enseignement et de recherche français ou étrangers, des laboratoires publics ou privés. 


\title{
Le rapport au temps, à l'espace et au discours des voix d'un point de vue psychopathologique
}

\section{A relation between time, space and hallucinated voices: a psychopathological point of view}

\author{
I. Guigo-Banovic *, G. Gimenez, J.-L. Pedinielli \\ Centre de recherche en psychologie de la connaissance, du langage et de l'émotion (EA3273), équipe thématique 3 : émotion, raisonnement el \\ pathologie, 29, avenue Robert-Schuman, 13621 Aix-en-Provence cedex 01, France
}

\section{Résumé}

L'hallucination acoustico-verbale (voix) est ici appréhendée comme objet de discours en dehors de toute conception perceptive. Les résultats descriptifs, déjà publiés, sont repris et approfondis par l'étude de cas d'une patiente psychotique. Les relations qu'elle entretient avec les voix, le temps et l'espace sont analysées d'un point de vue discursif (en tant qu'histoire hallucinée) puis psychopathologique (en référence au modèle psychanalytique). L'hallucination dans la psychose est appréhendée dans ce travail comme une tentative de gestion des tensions intrapsychiques. Elle figure l'impensé et permet la mise en dépôt de la tension qui n'a pu être gérée par le sujet.

\begin{abstract}
Voices are considered in this paper as subject of speech without perceptive conception. Descriptive results, already published, are deepe ned with a study case of a psychotic woman. The links she has with voices, time and space are examined as hallucinated stories then as a psychopathological point of view. This work attempts to set a paradigm of voices: hallucination in psychosis is a mean to manage intrapsychic tensions. It is a representation of unthinkable and it contains the psychic tension that psychotic people is not able to transform. Hallucinations are often viewed as a perception disorder or a cerebral dysfunction. DSM-IV considers them as a central symptom of schizophrenia. This paper, in a phenomenological approach, takes account auditory hallucinations (voices) as a clinical phenomenon and examines them as stories. These stories are studied with the narratives of psychotics patients. Voices are considered in this paper as subject of speech without perceptive conception. The methodology used is a discursive analysis of speech. Descriptive results, already published, are deepened with a study case of a psychotic woman. Discursive analysis focuses on the choice of common words of French language considered as indicators. It

permits a systematic descriptive analysis. The indicators are those of localization in time, in space and those of reported speech. The study c ase completes these processes. Empirical results show that psychotics are able to control their speech to an aim. The indicators of localization in time and space are linked with voices. Reported speech shows that the hallucinated speech must be told as it was produced by voices: there is no transformation. Study case of a psychotic woman describes the links she has with voices, time and space. They are examined as halluci-nated stories then as a psychopathological point of view. This work attempts to set a paradigm of voices: hallucination in psychosis is a mean to manage intrapsychic tensions. It is a representation of unthinkable and it contains the psychic tension that psychotic people is not able to transform.
\end{abstract}

\footnotetext{
* Auteur correspondant. Centre de recherche en psychologie de la connaissance, du langage et de l'émotion (EA3273), équipe thématique 3 : émotion, raisonnement et pathologie, Les-Jardins-de-Montfleury B, 276, chemin de la Calade, 83000 Toulon, France.

Adresse e-mail : ingrid.guido-banovic@wanadoo.fr (I. Guigo-Banovic).
} 


\section{Introduction}

Depuis l'Antiquité, l'hallucination acoustico-verbale (HAV) a suscité une production théorique massive. Alors que sa fréquence en fait le symptôme le plus habituel de la schizophrénie, sa définition n'est pas encore tranchée $[1,21,23]$. De nombreux courants s'opposent. Pour certains, peut-être les plus nombreux, l'HAV est un trouble de la perception, H. Ey [11] les ayant définies comme des perceptions sans objet à percevoir. Ils s'opposent ainsi aux auteurs qui la considèrent comme faisant partie des troubles du langage $[2,5,10,22]$. Au-delà de ce qu'elle pourrait être, d'autres s'interrogent sur sa fonction $[20,26-28]$ et son sens allant à l'encontre des courants, qui ne veulent y voir qu'un trouble du fonctionnement cérébral [24].

Le but de cette recherche est de s'interroger sur la manière dont il est possible de traiter la question de l'hallucination sans se référer à une définition marquée par le primat de la perception. Ce travail les considère d'abord comme un mode de rapport au discours : on passe alors d'une conception des voix en tant que symptômes à une conception des voix en tant qu'objet de discours. Elles ont alors une fonction dans la dynamique de communication avec un tiers puisqu'elles deviennent l'objet d'un récit. Cette perspective s'inscrit dans la démarche de J. Naudin [26,27] et de A. Tatossian [30] où il est nécessaire d'opérer un retour au fait clinique et de penser les hallucinations comme des histoires.

Le récit qui découle de cette expérience reflète un vécu, un éprouvé de manière immédiate et irréductible. La narration inscrit un événement dans un lieu et un temps partagés. Le narrateur situe et se situe par rapport à un événement qui fait rupture dans son existence. Par conséquent, quelle inscription spatiotemporelle cette expérience peut-elle entraîner dans le récit du sujet où le lieu de l'hallucination est avant tout le corps [18] ? Quels liens le sujet qui entend des voix entretient-il avec le temps, l'espace et le discours des voix ?

\section{Méthode}

Le discours a été le seul matériel étudié car il constitue l'unique moyen d'accéder à une expérience qui sans lui demeurerait impénétrable [8]. L'analyse de contenu, reposant sur le choix de mots de la langue française prenant alors valeur d'indicateurs, offre la possibilité d'une analyse descriptive systématique de l'ensemble des récits à notre disposition quelle que soit leur origine. L'étude de cas vient compléter cette démarche en prenant en considération les liens qui peuvent se dégager entre des résultats empiriques et le point de vue psychopathologique [31].

Les résultats empiriques $[3,4,17]$ montrent que les sujets psychotiques sont en mesure d'organiser leur discours dans un but précis. Les marqueurs de localisation dans le temps montrent que si les voix hallucinatoires ont une temporalité qui leur est propre, elles s'inscrivent aussi dans un rapport au temps qui est relatif à la personnalité du sujet qui raconte. Les marqueurs de localisation dans l'espace, eux, montrent que la référence n'est pas le sujet mais les voix qui constituent une référence spatiale organisatrice. Et, qu'elles soient intérieures ou extérieures au sujet, elles sont distinctes de lui et constituent à elles seules un point de référence dans l'espace.

Enfin, le discours rapporté le plus utilisé par l'ensemble des sujets était le discours rapporté cité. Il reproduit tel qu'il a été énoncé le propos tenu : ce que dit la voix ne peut être rapporté qu'au style direct, dans son intégrité énonciative initiale. L'emploi du discours rapporté indéterminé souligne la difficulté pour l'interlocuteur à démêler s'il s'agissait d'un discours produit dans l'ici et maintenant de l'actualité énonciative (les voix parlent « en direct») ou s'il s'agissait d'un discours rapporté cité difficilement repérable en tant que tel.

\section{Mai Li}

Mai Li est une patiente d'origine vietnamienne âgée de 45 ans, dont la famille s'est exilée en France il y a de nombreuses années. Le diagnostic posé est celui d'une schizophrénie à début tardif ou d'une psychose hallucinatoire chronique. Hospitalisée depuis presque un an au moment de l'entretien, elle évoque l'assassinat de son père au Viêt-nam pour des raisons politiques. Mai Li dit être la victime de personnes malveillantes qui la volent, la surveillent, l'épient. Elle entend la voix d'un homme depuis plusieurs décennies et elle l'identifie comme étant celle d'un étudiant éconduit qui se prénomme Patrick.

\subsection{Le rapport au temps}

Mai Li construit un récit dont le passé demeure très proche du présent. Elle établit un lien vivant entre le passé et le présent : « [...] Elle [la voix] a dit qu'elle fera tout pour me nuire. Les vols, mes papiers qu'elle a volés, la constitution du dossier médical que j'avais fait, mes bulletins de paie, mes talons de chéquier, mes bijoux, mes fourrures, mes vêtements, tout, tout. Le studio, j'ai vidé le studio ; maintenant mes derniers habits que j'ai achetés, je les ai mis chez mes parents. [...] Tout ce que je mets dans mon studio il disparaît et à Paris c'était pire. » Elle va relier des événements indépendants les uns des autres dans une perspective de mise en sens intrinsèquement liée à cette voix d'homme : « [...] C'est cet homme qui m'a poursuivie et pendant toutes ces années qui m’a volé mes affaires, qui m'a fait accuser ma famille, 
mes cousins, mes amants. Tous, je les ai traités de voleur alors qu'ils ont avoué n'avoir rien fait. C'était un étudiant qui était fou amoureux de moi qui me poursuivait en $4 \mathrm{~L}$ d'Aix à Marseille, qui était en DS noire, il ne bougeait pas, il ne décollait pas de devant ma chambre. » Des affaires de Mai Li sont perdues, elle en fait le constat et en cherche la cause : elles lui ont été volées. Des proches sont, dans un premier temps, accusés (parents, cousin) puis des relations intimes (amants) et enfin un homme qu'elle ne connaît que de vue (l'étudiant anonyme) mais qui aurait voulu devenir son amant. Ce récit met en évidence que son actualité (puisqu'on la vole toujours) est reliée à un passé lointain (à l'époque où elle était étudiante). Conjointement à ce rapprochement temporel, on peut remarquer une mise à distance de l'implication des proches à ces vols : du cercle de la famille, les soupçons se dirigent vers les amants pour finalement se cristalliser autour d'un étudiant anonyme. Tout va alors s'éclairer : Mai Li a à peine remarqué cet étudiant qui, se sentant alors éconduit, se venge en la volant. Il se vengera encore d'elle puisque sa voix dit explicitement qu'elle ne va pas guérir.

L'organisation temporelle dans la construction du récit est cohérente, mais on peut observer une distorsion de ce que peuvent représenter passé et présent, le noyau organisateur étant la découverte du supposé désir de cet étudiant (par la suite qualifié de malveillant).

\subsection{Le rapport à l'espace}

Le rapport à l'espace dans le récit de Mai Li s'énonce dans une perspective arrière : Patrick la poursuit. Il est derrière elle pour l'épier, il la tourmente et l'obsède. La position derrière elle et le tourment sont mis en équation. Mai Li raconte alors les circonstances d'une hospitalisation assez ancienne : «[...] après le travail mon manager m'a demandé si je voulais rentrer à la maison parce que j'étais fatiguée. Il m'a emmenée à l'hôpital au lieu de m'emmener chez moi. Il m'a laissée là et des infirmiers m'ont prise et m'ont emmenée dans un hôpital psychiatrique dans le troisième. Je crois que c'était dans le troisième. Là-bas on m'a déshabillée et j'avais une blouse blanche ouverte là derrière, sans culotte, sans rien et je me suis échappée de là et personne n'était au courant [...]. » Des hommes en habit l'ont déshabillée puis revêtue partiellement laissant à nu la partie postérieure de son corps. La mise à découvert de cette partie du corps renvoie pour Mai Li à une incapacité à se défendre contre ce qui pourrait survenir par-derrière. Elle traite ce qui est métaphorique (être prise par-derrière, se dévoiler, se mettre à nu) au pied de la lettre : elle est mise nue et ce qu'elle cherchait à cacher est dévoilé (sa souffrance, son intimité); elle est donc mise à nu : les mots sont traités comme des choses [21] et l'expression est appréhendée de façon démétaphorisée [22]. Elle précise que même si elle porte un vêtement celui-ci est ouvert dans le dos et laisse voir son derrière. À moitié habillée, avec une souffrance qui ne peut plus être cachée, Mai Li ne peut que se sentir impuissante, dans l'incapacité à se défendre. La thématique sexuelle anale est ici évidente : Mai Li ne peut se défen- dre contre ce qui pourrait surgir par-derrière/par le derrière, et Patrick est à sa poursuite (il est derrière elle). Il constitue donc une menace pour son intégrité corporelle qui, à ce moment précis, est justement mise en position de faiblesse. Patrick est un amoureux éconduit qui pourrait vouloir prendre à revers ce qui lui est refusé. La thématique sexuelle resurgit de manière brutale et menaçante. Elle s'échappe de l'hôpital, dit-elle, personne n'est au courant. En se sauvant, en échappant à tous ces hommes, elle préserve son intimité physique et psychique et reprend le dessus. Elle recouvre le contrôle : elle fait face. Elle exprime son état interne en termes figurés : la position de face se retrouve dans le fait que Patrick est devant chez elle, qu'il s'est trouvé devant elle au restaurant universitaire. Ils se font donc a priori physiquement toujours face. On peut donc se demander si ce n'est pas plus l'idée qu'il la poursuive, qu'il soit à ses trousses qui l'effraye, que de se trouver face à lui. Et, être derrière implique la notion de passé. Mai Li n'a aucune maîtrise possible sur ce qui appartient au passé ; en revanche, elle pourrait contrôler ce qui l'attend devant, dominer l'avenir en donnant du sens après coup au passé. Non seulement ce qui spatialement est dans le dos, l'idée d'être poursuivie se trouvent être reliés temporellement avec ce qui, de fait, est derrière elle : à savoir les événements de sa vie, ses souvenirs.

Mai Li raconte qu'on la vole sans cesse, qu'on lui dérobe tout ce qui constitue son intérieur. C'est l'intérieur domestique qui est dérobé et c'est l'intérieur psychique qui est pris en otage. Il y a une perte de «substance », faisant passer de quelque chose de construit, de plein, à une notion de vide, d'espace plat, sans relief. Mai Li explique qu'au départ elle pensait que la voix provenait de ses boucles d'oreilles puis de ses plombages, pour enfin la localiser dans sa tête. Il y a donc à la fois un mouvement progressif de l'extérieur vers l'intérieur et mise en relation d'un objet extérieur (un micro) à un objet qui se dissout pour agir dans le corps. Le fait qu'elle ne reconnaisse plus ses pensées comme étant les siennes s'accompagne d'une attribution de celles-ci (qui deviennent alors des voix) à une origine extérieure (xénopathie). Et cette source dans un premier temps prend une caractéristique matérielle puisque c'est un micro. Puis elle va perdre de plus en plus de sa substance. Ce qui était au départ une pensée retrouve progressivement (via l'extérieur, une matérialisation puis une dissolution) une place dans son univers privé. Mais elle ne retrouve pas ce caractère silencieux et personnel en tant que tel. De plus, ce mouvement de l'extérieur vers l'intérieur s'accompagne d'une perte progressive du contrôle sur la voix et sur sa pensée. Et, plus la perte de contrôle devient flagrante, plus elle aura tendance à projeter la source de cette voix à l'extérieur. Ce ne sont pas ses pensées qu'elle entend, c'est une voix qui vient du dehors et qui chemine, s'installe progressivement dans sa tête. Mai Li pense que la raison de cet «empoisonnement » serait qu'elle aurait failli dans son militantisme politique (elle était membre actif au sein d'un parti de droite). Le clivage politique droite-gauche s'amenuise : la gauche lui veut du mal car c'est une «bonne » militante, et la droite ne l'aide pas à se débarrasser de cette 
voix car elle aurait failli (au moment d'une défaite aux élections législatives). Il y a donc un rapprochement des deux parties en un tout hostile via la voix. Le corps politique n'est plus scindé, il est uni ; ce qui lui laisse entendre qu'alors son propre corps est lui aussi menacé. Mai Li dit : « [...] J'avais tellement peur, je me suis dit si je continue ils vont s'en prendre à ma famille et tout, parce que, il faut, il faisait, la voix ne faisait que menacer ma famille et j'ai un frère qui à l'époque était à Paris avec moi. On lui a fait des rayons laser, il est styliste de mode, il a les doigts des mains recourbés et il pouvait plus bouger du poignet. Je me suis dit c'est la gauche qui a dû nous punir et j'ai un autre frère à qui on a opéré des yeux et il est mal voyant maintenant. C'est à l'époque où j'ai milité que tous ces événements se sont produits dans ma famille. » Elle seule ne semble pas être encore atteinte physiquement. Elle se sent menacée mais pas encore détruite. L'engagement politique d'une femme de la famille fait courir une menace corporelle sur les hommes. Pour elle, même si la voix est dans la tête, qu'elle parasite ses pensées, le choix de l'atteindre est plus psychique que physique. Elle décrit un phénomène où ses idées deviennent dangereuses pour elle et pour les autres. Mai Li doit les cacher (elle taît son engagement à sa famille) puis elle les perçoit comme source de danger. Elles menacent son intégrité psychique en la mettant face à un conflit insoluble : elles la mettent en péril psychiquement et portent atteinte à l'intégrité physique des hommes de la famille.

\subsection{Le discours rapporté}

Le discours de la voix est devenu une habitude, un stéréotype car on ne repère pas de grande variation dans le contenu. La voix lui dit : «Faut pas espérer guérir, vous serez toujours malade, et vous serez toujours malade, et vous serez toujours hospitalisée euh... je ne tiens pas à ce que vous guérissiez quoi. » Cette voix l'insulte sur un thème apparemment récurrent : «Si elle m'insulte. Elle me dit que je suis une salope parce que je n'ai pas voulu de lui et que j'ai eu plein d'amants, et, que, pourtant il n'était pas manchot [...]. » Deux hypothèses s'ouvrent à nous. La première serait qu'au fur et à mesure du temps qui passe, Mai Li conserverait plus l'idée que la forme de ce qui lui est dit. Il y a une certaine logique d'intégration du discours de la voix à sa propre trame discursive. La seconde hypothèse s'oppose à la première : c'est ce qui pourrait lui être dit qui est princeps, puis, vient à certains moments la verbalisation hallucinée. Les deux pistes sont cohérentes et ne sont pas contradictoires, elles relèvent de deux logiques différentes. L'une va dans le sens du récit où le temps qui passe, qui défile, abrase la forme tout en préservant le contenu. L'autre renvoie au processus de manifestation de la voix hallucinatoire où l'idée vient se figurer sous l'apparence d'une perception.

La thématique sexuelle (au-delà de la thématique anale) est clairement évoquée puisque c'est un homme «pas manchot » qui lui parle. Elle évoque ses amants mais la place centrale est tenue par un homme qui ne l'a pas été. Beaucoup d'hommes l'ont semble-t-il courtisée, comblée de cadeaux de valeur, etc. Et le premier homme qu'elle accuse de l'avoir volée est son cousin qui est, a-t-elle besoin de préciser, homosexuel. Pour Mai Li, être un homme poserait question. En effet, si nous reprenons l'histoire de Mai Li, la voix qu'elle entend serait apparue en 1986 au moment des élections législatives. L'engagement politique qu'elle a tenu secret à sa famille pendant longtemps est un fil conducteur qui la rapproche de son père. Elle raconte brièvement que son père, militant lui aussi, était engagé et qu'il aurait été assassiné pour des raisons politiques. Un complot serait ourdi afin de lui nuire comme cela a été le cas pour son père. Il y a donc des tractations secrètes contre elle dans les plus hautes sphères de l'État (c'est ce qu'elle sous-entend à la fin de l'entretien en disant que la droite et la gauche se sont liées pour lui nuire). Des propos sont échangés de manière masquée. La question d'un discours se pose aussi ici. La politique rapportée à son père renvoie directement à l'autorité et à son exercice. Les hommes politiques exercent une autorité sur l'État. Son père faisait de la politique et par conséquent voulait peutêtre avoir une certaine supériorité dans la société comme il pouvait avoir autorité sur sa fille. Aujourd'hui, celui qui a autorité sur elle est un homme presque anonyme qui n'est ni un amant, ni un homosexuel, ni un être castré. La question de la différence des sexes se pose alors à elle dans ces termes. Être engagée politiquement, faire entendre sa voix (au sens propre comme au figuré) dans la lutte pour le pouvoir entraîne obligatoirement d'être menacée de mort, d'être tout au moins l'objet d'un complot ayant pour but minimum de lui nuire.

L'étude du récit de Mai Li a permis de décrire les liens étroits qu'entretiennent le temps et l'espace. L'espace s'organise comme le temps : ce qui est derrière correspond au passé, devant au futur, et ici au présent. Les notions de poursuite et d'affrontement sont liées à la position spatiale de l'homme qui la poursuit, qui est dans son dos.

Le rapport du sujet au discours de la voix a montré que la parole de l'un barrait la route à la parole de l'autre. Non seulement il y a une relation d'autorité, de subordination mais aussi un effacement partiel des barrières corporelles. Il n'y a donc plus de confrontation matérielle entre deux instances énonciatives intégrées à un corps, mais confrontation d'au moins deux instances énonciatives mises en concurrence.

\section{Discussion psychopathologique}

Dans la schizophrénie [6], le temps ne s'écoule plus, i] s'agglutine dans un présent où le passé n'est jamais complètement révolu et l'avenir difficilement envisagé. Nous sommes ici proche de ce que Maldiney [25] nomme la «surproximité compacte » observée chez les schizophrènes, dans le temps et l'espace, qui marque l'immense difficulté à prendre de la distance.

Dans le récit de Mai Li, passé et présent existent mais le futur n'est pas employé : il est envisagé de manière détournée via des expressions relatives à des positions corporelles. 
Le déroulement du temps se fige autour du seul présent. Passé et futur ne s'articulent pas, ils sont « court-circuités ». Les phénomènes hallucinatoires ont une charge émotionnelle et psychologique très forte, il n'y a plus de flux, de mouvement dynamique. Le rapprochement d'événements passés avec le présent entraîne un enchaînement en terme de causalité directe.

Le fait que la voix soit localisée derrière le sujet entretient le sentiment de persécution et renvoie à la thématique sexuelle insupportable. Elle est intolérable car elle implique une remise en cause des barrières corporelles et surtout l'impossibilité de maitriser ce qui se passe derrière. Lorsque les voix sont localisées en face, il y a un arrêt de la fuite, une tentative de maîtrise de ce qui survient [29]. Il y a dans les deux cas un traitement au sens propre de ce qui pourrait être aussi compris au sens figuré. Enfin, lorsque l'idée d'être devant est soulevée, c'est le futur qui est envisagé, comme si le sujet devait les affronter de nouveau.

La perspective tridimensionnelle existe mais elle est vite mise en concurrence avec la perspective plane. En effet, la tête est envahie, le corps traversé voire même transpercé par des ondes. Si un intérieur peut être désagrégé, si un corps peut être habité, c'est justement parce que la notion de volume existe. Mais, la notion de perte de substance, l'incapacité à déterminer si les phénomènes hallucinatoires viennent de l'extérieur ou de l'intérieur mettent en concurrence une perspective plane, topographique. Comme si les voix traversant les barrières corporelles annihilaient la perspective tridimensionnelle du sujet schizophrène. Mais cette notion de volume va être conférée aux voix elles-mêmes. Qu'elles soient localisées à l'intérieur ou à l'extérieur de la tête, elles n'en sont pas moins considérées comme distinctes du sujet, ce qui renforce leurs caractéristiques xénopathiques. Elles sont donc étrangères, distinctes du sujet qui parle mais surtout la mise en récit leur confère un corps : l'expérience hallucinatoire elle-même leur confère un espace et une forme, puis, seulement après, la mise en parole, adressée à un autre, leur donne une autre dimension. Et la voix reprend à son propre compte le volume, l'aspect tridimensionnel qui va alors manquer au sujet. Le schizophrène s'efface, et comme le décrit Bion [7], ne devient qu'un point alors que la voix s'habille, revêt comme un habit le corps alors abandonné. Il y a mise en évidence d'une certaine forme de projection venant de l'intérieur vers une réalité extérieure mal différenciée. L'espace prend une tonalité différente : les voix sont des entités autonomes aux caractéristiques diaboliques qui confèrent ces qualités à l'espace [6].

Binswanger rapporte dans un cas de schizophrénie la manière dont son patient conçoit ses hallucinations : « [...] Pour désigner tout cet espace déterminé par l'unité de sens du démoniaque, le malade a l'expression spatiale caractéristique d'une "salle d'audience". Cette salle d'audience sert de base à un "orateur d'une autorité particulière" ; elle représente "une discussion ouverte sous un aspect particulier". [...] » [6]. Les voix entretiennent donc une relation d'autorité dans un temps et un espace caractéristiques. De plus, la parole de l'un barre la route à la parole de l'autre. Non seulement il y a une relation d'autorité, de passivité mais aussi un effacement des barrières corporelles. Il n'y a plus de confrontation matérielle entre deux instances énonciatives intégrées à un corps, mais confrontation d'au moins deux instances énonciatives mises en concurrence. Intérieur et extérieur se rejoignent dans ce discours halluciné qui va être appelé et rappelé. Les voix viennent alors figurer à l'extérieur sous une modalité analogique à la perception ce qui a été énoncé à un moment donné du développement psychique et qui n'a pu être introjecté [16]. La polyphonie intérieure insuffisamment constituée et intégrée trouve ce qui lui fait défaut dans une voix projetée à l'extérieur. Une comparaison avec l'anamorphose peut mettre en forme une idée beaucoup plus globale du fonctionnement psychique étudié.

L'anamorphose est un dessin distordu qui, vu dans un miroir cylindrique, reprend sa forme réelle. Il s'agit d'un effet pictural qui consiste à déformer un motif qui vu sous un certain angle reprend son aspect véritable. Ici, le thème serait la polyphonie qui se figure par la voix entendue. Cette polyphonie demeure déformée chez le schizophrène alors qu'elle reprendrait sa forme réelle, son aspect véritable chez le sujet non psychotique. Cela fait retour via l'extérieur [13,14], accompagné d'une menace terrible d'explosion interne, de perte de contrôle de la pensée sous la forme d'une injonction, d'une relation de subordination comme tout individu est subordonné à cette règle sociale et biologique. Ce discours universel est intrusif car il génère une angoisse massive d'éclatement, d'impossibilité à acquérir une unité subjective. Et dans le même temps, il ne peut être que constitutif car il conduit à une différenciation et à une autonomisation du sujet.

Dans la théorie freudienne, ce qui est insupportable est rejeté et revient du dehors, appréhendé comme une perception [12,13]. Or C. et S. Botella [9] vont aller plus loin dans la conception de ce qui est sous-jacent à l'hallucination psychotique. Pour eux est importante : « [...] la notion freudienne de l'abolition au-dedans des représentations qui ensuite reviendraient de l'extérieur ; puis Lacan, prolongeant Freud, apporte avec sa notion de forclusion l'explication des hallucinations psychotiques. [...] on ne peut comprendre l'hallucination psychotique qu'encadrée dans une dynamique d'ordre général : représentation, perception, hallucination. [...] toute hallucination doit être considérée comme un processus régrédient de la psyché. Elle devient psychotique quand cette régrédience dépasse un certain degré, une certaine borne. La forclusion se présente quand cette régression va jusqu'aux investissements de la représentation de chose inconsciente de l'objet. Celle-ci est "négativée", abolie dit Freud [...]. »

Cette protection contre le morcellement que réactive la polyphonie, le schizophrène va la trouver dans l'hallucination via une régression qui ramène aux investissements de la représentation de chose inconsciente de l'objet [19].

À la régression va se joindre un mécanisme de défense, la projection. Elle va alors amener le sujet à localiser à l'extérieur ce qui est intolérable à l'intérieur. Les liens qui existent entre les schizophrènes et les voix qu'ils entendent sont com- 
plexes : il y a en même temps attirance et répulsion, confiance absolue et foncière méfiance.

L'hallucination dans la psychose est un moyen, une tentative de gestion des tensions intrapsychiques. Elle protège le sujet du morcellement et elle le fait sous le mode de la décharge $[13,15,16]$. L'hallucination transforme le monde externe [14] afin de le rendre plus supportable et elle utiliserait non pas des représentations de mots mais des représentations de choses. Enfin, d'un point de vue génétique, l'hallucination est une régression. Nous sommes là d'accord avec ce que dit C. Botella (ibid.) : «elle devient psychotique lorsque cette régression va jusqu'aux investissements de la représentation de chose de l'objet. » La polyphonie constitutive est réactivée et fait retour grâce à la projection dans le registre du même. Dans la psychose, et à cause d'elle, l'hallucination figure (et renferme) l'impensé [13], et permet la mise en dépôt de la tension qui n'a pu être gérée par le sujet. La polyphonie est projetée par anamorphose et fait retour par le maintien de l'identité de perception.

\section{Conclusion}

Le retour au fait clinique et à l'étude des récits de patients hallucinés a permis de mettre en lumière le rapport discursif que peut entretenir le patient avec ses voix. Il a aussi rendu possible l'inscription de l'approche discursive dans une réflexion psychopathologique où les voix sont pensées et conçues comme des histoires.

\section{Références}

[1] American Psychiatric Association. Diagnostic and Statistical Manuel of Mental Disorder. 4th edition. Washington: APA; 1994.

[2] Andreasen N. Thought, language and communication disorders. Arch Gen Psychiatry 1979;36:1315-34.

[3] Banovic I. Contribution à l'étude discursive des récits de l'expérience subjective des voix : temps, espace et discours rapporté. Thèse de Doctorat. Nouveau Régime en Lettres et Sciences Humaines sous la direction du Professeur Jean-Louis Pedinielli. Université de Provence (Aix-Marseille 1), 2001.

[4] Banovic I. Une étude préliminaire sur le repérage des voix dans le discours grâce à des marqueurs de localisation dans le temps, dans l'espace, et des marqueurs de polyphonie. Ann Med Psychol (Paris) 1999;157:650-4.

[5] Barch D, Berenbaum H. Language production and thought disorder in schizophrenia. J Abnorm Psychol 1996;5:81-8.
[6] Binswanger L. Le problème de l'espace en psychopathologie. 1932 Préface et traduction C. Gros-Azorin. Toulouse: Presses Universitaires du Mirail; 1998.

[7] Bion WR. Transformations. 1965. Paris: Presses Universitaires de France; 1982.

[8] Blanchet A, et al. Recherches sur le langage en Psychologie clinique. Paris: Dunod; 1997.

[9] Botella C, Botella S. La problématique de la régression formelle de la pensée et de l'hallucinatoire. In: Revue Française de Psychanalyse, Colloque S.P.P. Unesco du 14 et 15 janvier. 1989. p. 63-90.

[10] Davies P, Thomas P, Leudar I. Dialogical engagement with voices: a single case study. Br J Med Psychol 1999;72:179-87.

[11] Ey H. Traité des hallucinations, 1.2. Paris: Masson; 1973.

[12] Freud S. Métapsychologie. 1915. Paris: Gallimard; 1968.

[13] Freud S. Remarques psychanalytiques sur l'autobiographie d'un cas de paranoïa. (Le Président Schreber). 1911. Cinq Psychanalyses, 263-324. Paris: PUF; 1992.

[14] Freud S. Névrose, Psychose et Perversions. 1924. Paris: PUF; 1973.

[15] Gimenez G, Pedinielli JL, Guimont M. La démétaphorisation dans le travail de l'hallucination. Revue française de psychiatrie et de psychologie médicale 1999;32:50-3.

[16] Gimenez G. Clinique de l'hallucination psychotique. Paris: Dunod; 2000.

[17] Guigo-Banovic I, Naudin J, Collet R, Pedinielli JL. Le rapport au temps et à l'espace dans les récits de l'expérience subjective des voix. Ann Med Psychol (Paris) 2003;161:774-9.

[18] Jover F. Esquisse d'une topographie hallucinatoire. In: Introduction à la phénoménologie des hallucinations, sous la direction de G. Charbonneau. Collection Pheno; 2001. p. 43-60.

[19] Lacan J. Les psychoses. Le Séminaire, livre III. 1955-56. Paris: Seuil; 1981.

[20] Lazorthes G. Les hallucinations. Paris: Masson; 1997.

[21] Leudar I, Thomas P, Johnston M. Self repair of schizophrenics: effects of hallucinations and negative symptoms. Brain Lang 1992;43:487511.

[22] Leudar I, Thomas P, Mc Nally D, Glinski A. What voices can do with words: pragmatics of verbal hallucinations. Psychol Med 1997;27: 885-98.

[23] Liester MB. Inner voices: distinguishing transcendent and pathological characteristics. The Journal of Transpersonal Psychology 1996;28: $1-30$.

[24] Liester MB. Toward a new definition of hallucination. Am J Orthopsychiatry 1998;68:305-12.

[25] Maldiney H. Penser l'homme et sa folie. Grenoble: Million; 1997.

[26] Naudin J. Phénoménologie et Psychiatrie. Les voix et la chose. Toulouse: PUM; 1997.

[27] Naudin J, Pringuey D, Azorin JM. Approche phénoménologique des hallucinations. Les Hallucinations, sous la direction de D. Widlöcher. 2002.

[28] Pedinielli JL, Bertagne P, Von Kracht H. Paroles de psychotiques. Nervure 1990;3:10-5.

[29] Rosolato G. Les hallucinations acoustico-verbales et les champs perceptifs du corps. Evol Psychiatr (Paris) 1977;XLII:729-41.

[30] Tatossian A. Phénoménologie des psychoses. 1979. L’Art du Comprendre. 1997.

[31] Widlöcher D. Le cas, au singulier. Nouvelle Revue de Psychanalyse 1990;42:285-302. 